\title{
Residencia médica en Alemania: una opción de excelencia abierta para médicos mexicanos
}

\author{
Edgar Santos ${ }^{1}$, Humberto Silos², Arturo Olivares-Rivera ${ }^{1,2}$, Valente Fragosa-Padilla ${ }^{3}$ \\ y Renán Sánchez-Porras ${ }^{1,4}$ \\ ${ }^{1}$ Departamento de Neurocirugía, Universidad de Heidelberg, Heidelberg; ${ }^{2}$ Departamento de Cirugía General, Schön Klinik Nürberg-Fürth, Nürnberg; \\ ${ }^{3}$ Neurosurgical Practice Lake Constance, Singen; ${ }^{4}$ Departamento de Neurocirugía, Hospital Ludwigsburg, Ludwigsburg. Alemania
}

Alemania es una de las mayores economías de Europa, y tiene una indiscutible influencia política, científica y tecnológica. Posee 82 millones de habitantes y dispone de un sistema de salud con cobertura total, el cual es costeado por casas aseguradoras tanto públicas como privadas. En contraste, en México, de acuerdo con la Organización Mundial de la Salud (OMS), hay un desarrollo ambivalente en el espectro de enfermedades causantes de un número mayor de muertes, por un lado común en los países desarrollados, un aumento de enfermedades relacionadas con el consumo y la vida sedentaria que causan obesidad, y enfermedades como la diabetes mellitus, cardiopatías y arterioesclerosis en la población de edad adulta, y por otro lado enfermedades comunes a países tercermundistas, que tienen que ver con la falta de cobertura de servicios médicos básicos, agua potable, sistema de recolección de basura, falta de red de alcantarillado y tratamiento de aguas negras, provocando enfermedades gastrointestinales graves, sobre todo en la edad infantil, mientras que el agravio de las diferencias sociales y la inseguridad económica propician un aumento de muertes por causas violentas en el segmento de la población joven.

No solo existe un estancamiento en el sector salud, el sector socioeconómico y el sector meramente económico, sino también en el sector de ciencia y tecnología, siendo que el gobierno y sus estructuras no permiten la simple cobertura médica esencial en la población y mucho menos avances en el sistema educativo en México, donde hay un excedente de recursos humanos poco cualificados y poca oferta de trabajo, favoreciendo el desarrollo de condiciones de trabajo desfavorables para el empleado. Esto trae consigo un aumento en el deseo de cada vez más profesionistas de excelencia de desarrollarse en el extranjero. Actualmente, en Alemania existe un déficit de profesionistas de la salud. Las actuales reformas laborales en el sistema de salud limitaron las jornadas de trabajo de los médicos, incrementando la necesidad de emplear mayor número de personal cualificado para cubrir de manera cabal las jornadas laborales. A esto se suma el aumento en el número de médicos en proceso de jubilarse, lo que ha dado origen a una apertura para que muchos médicos extranjeros laboren en Alemania. Con esto, el gobierno se vio en la necesidad de abrir sus fronteras y facilitar la convalidación de estudios incluso a ciudadanos de países no europeos.

De acuerdo a las estadísticas de la Cámara Nacional de Médicos de Alemania (Bundesärztekammer), este país tiene un total de 378,607 médicos, de los cuales 46,721 son de origen extranjero. La mayoría de los médicos extranjeros provienen de Rumanía (4285), Grecia (3118) y Siria (2895). México como tal no aparece como entidad autónoma en las estadísticas, sino en conjunto en el rubro de «médicos extranjeros de Norteamérica», sumando 404 (http://www. bundesaerztekammer.de/ueber-uns/aerztestatistik/ aerztestatistik-2016/).
Fecha de recepción: 01-06-2017

Fecha de aceptación: 12-06-2017

DOI:10.24875/GMM.17003486
Gac Med Mex. 2017;153:514-515

Contents available at PubMed www.gacetamedicademexico.com 


\section{Puntos clave de interés en el sistema alemán}

- Sistema hospitalario: en el país distinguen diversos tipos de hospitales; los hay universitarios (considerados como la opción de mayor prestigio), regionales, comunitarios, privados y clínicas de rehabilitación. Ello permite una variabilidad en la elección del hospital para trabajar de acuerdo al perfil académico del aplicante.

- Condiciones laborales: en términos generales, las condiciones laborales incluyen una jornada de 40 horas semanales, las cuales, en la mayoría de los casos. no se logra respetar; sin embargo, el tiempo extra producido es compensado, ya sea con tiempo libre o con el pago de horas extra. Además, se cuenta con alrededor de 30 días hábiles de vacaciones al año y 3 días para congresos.

- Trabajo clínico y educación: a diferencia del sistema de residencias mexicano, la residencia médica en Alemania es vista como un trabajo. No existe una distinción jerárquica marcada entre los años de residencia. El trabajo es muy individualizado y se trabaja de manera estrecha con el adscrito. En las guardias suele estar un solo residente a cargo del departamento, con un adscrito disponible por teléfono. La formación médica es muy autodidacta y suele ser orientada a la práctica, basada en el principio de «aprender haciéndolo». Además, existe la oportunidad de hacer investigación, la cual se lleva a cabo en su mayoría en los hospitales universitarios. Los médicos que hacen ciencia tienen más puertas abiertas y reciben más y mejores ofertas de trabajo.

- Pago: los sueldos de los residentes y especialistas están establecidos por ley en el ámbito nacional, y pueden consultarse de manera pública (http://oeffentlicher-dienst.info/aerzte/). En términos generales, van de 2500 a 10,000 euros al mes.

\section{Oportunidad para médicos mexicanos}

Las probabilidades para que un médico mexicano emigre están principalmente en relación con las posibilidades económicas, las condiciones laborales y la capacidad para superarse. No obstante, ¿qué se requiere para ingresar al sistema de salud alemán? Se puede consultar en esta liga: http://www.bundesaerztekammer.de/aerzte/internationales/medizinstudium-und-aerztliche-taetigkeit-in-deutschland/. De manera oficial, se requieren como puntos básicos:

- Idioma alemán, nivel C1.

- Estudios de Medicina en México en una universidad reconocida por la OMS.

- En algunos Estados, acreditar el conocimiento de medicina por medio de un examen de homologación (Kenntnisprüfung).

- Oferta de trabajo de algún hospital (no siempre es requisito).

- Demás documentos oficiales y traducidos al alemán por una institución reconocida por la Unión Europea

No se requiere examen nacional de residencia. El hospital en particular escoge a los residentes dependiendo del currículo y sobre todo por el nivel de alemán, los antecedentes de prácticas hechas en hospitales alemanes y la experiencia laboral en Alemania con pacientes alemanes.

\section{Beneficios para México}

Si bien estamos de acuerdo en que lo ideal no es que un intelectual deje su país, un talento mexicano tiene más dificultades en casa para encontrar las posibilidades de contribuir a su propio país y a la humanidad. Mientras tanto, países como China, India e incluso países europeos facilitan que sus mejores estudiantes emigren, pues si bien no pueden formar a todos en casa, sí tienen la visión de que, en un país con las condiciones adecuadas para su desarrollo, el estudiante puede convertirse en un referente internacional, revolucionar un área o crear nuevas áreas de conocimiento o industrias. Históricamente, también muchos de los mejores médicos mexicanos se han formado en el extranjero. En resumen, cuando un mexicano de excelencia llega a Alemania, México tiene beneficio de diferentes formas:

- Vínculo académico entre instituciones nacionales y extranjeras de calidad.

- Facilitación de intercambio de más personas.

- Transferencia de conocimiento y tecnología.

- Cooperación en proyectos transnacionales.

- Incremento del prestigio de México en el mundo.

- Regreso como fuerza de trabajo mejor cualificada.

- Mejoramiento del sistema de salud mexicano tomando ejemplo en el sistema de salud alemán. 